\title{
Strong convergence to a fixed point of a total asymptotically nonexpansive mapping
}

\section{Gang Eun Kim*}

\section{*Correspondence:}

kimge@pknu.ac.kr

Department of Applied

Mathematics, Pukyong National

University, Busan, 608-737, Korea

\begin{abstract}
In this paper, we prove strong convergence for the modified Ishikawa iteration process of a total asymptotically nonexpansive mapping satisfying condition (A) in a real uniformly convex Banach space. Our result generalizes the results due to Rhoades (J. Math. Anal. Appl. 183:118-120, 1994).
\end{abstract}

MSC: $47 \mathrm{H} 05 ; 47 \mathrm{H} 10$

Keywords: strong convergence; fixed point; modified Ishikawa iteration process; total asymptotically nonexpansive mapping

\section{Introduction}

Let $X$ be a real Banach space, let $C$ be a nonempty closed convex subset of $X$, and let $T$ be a mapping of $C$ into itself. Then $T$ is said to be asymptotically nonexpansive [2] if there exists a sequence $\left\{k_{n}\right\}, k_{n} \geq 1$, with $\lim _{n \rightarrow \infty} k_{n}=1$, such that

$$
\left\|T^{n} x-T^{n} y\right\| \leq k_{n}\|x-y\|
$$

for all $x, y \in C$ and $n \geq 1$. T is said to be uniformly L-Lipschitzian if there exists a constant $L>0$ such that

$$
\left\|T^{n} x-T^{n} y\right\| \leq L\|x-y\|
$$

for all $x, y \in C$ and $n \geq 1$. If $T$ is asymptotically nonexpansive, then it is uniformly $L$-Lipschitzian. We denote by $\mathbb{N}$ the set of all positive integers. $T$ is said to be total asymptotically nonexpansive (in brief, TAN) [3] if there exist two nonnegative real sequences $\left\{c_{n}\right\}$ and $\left\{d_{n}\right\}$ with $c_{n}, d_{n} \rightarrow 0$ as $n \rightarrow \infty, \phi \in \Gamma\left(R^{+}\right)$such that

$$
\left\|T^{n} x-T^{n} y\right\| \leq\|x-y\|+c_{n} \phi(\|x-y\|)+d_{n},
$$

for all $x, y \in C$ and $n \geq 1$, where $R^{+}:=[0, \infty)$ and $\phi \in \Gamma\left(R^{+}\right)$if and only if $\phi$ is strictly increasing, continuous on $R^{+}$and $\phi(0)=0$. It is clear that if we take $\phi(t)=t$ for all $t \geq 0$ and $d_{n}=0$ for all $n \geq 1$ in (1.2), it is reduced to (1.1). Approximating fixed points of the modified Ishikawa iterative scheme under total asymptotically nonexpansive mappings has been investigated by several authors; see, for example, Chidume and Ofoedu $[4,5]$, Kim [6], Kim and Kim [7] and others. For a mapping $T$ of $C$ into itself in a Hilbert space, 
Schu [8] considered the following modified Ishikawa iteration process ( $c f$. Ishikawa [9]) in $C$ defined by

$$
\begin{aligned}
& x_{1} \in C, \\
& x_{n+1}=\left(1-\alpha_{n}\right) x_{n}+\alpha_{n} T^{n} y_{n}, \\
& y_{n}=\left(1-\beta_{n}\right) x_{n}+\beta_{n} T^{n} x_{n},
\end{aligned}
$$

where $\left\{\alpha_{n}\right\}$ and $\left\{\beta_{n}\right\}$ are two real sequences in $[0,1]$. If $\beta_{n}=0$ for all $n \geq 1$, then iteration process (1.3) becomes the following modified Mann iteration process (cf. Mann [10]):

$$
\begin{aligned}
& x_{1} \in C, \\
& x_{n+1}=\left(1-\alpha_{n}\right) x_{n}+\alpha_{n} T^{n} x_{n},
\end{aligned}
$$

where $\left\{\alpha_{n}\right\}$ is a real sequence in $[0,1]$.

Rhoades [1] proved the following results which extended Theorems 1.5 and 2.3 of Schu [8] to uniformly convex Banach spaces.

Theorem 1.1 Let $X$ be a uniformly convex Banach space, let $C$ be a nonempty bounded closed convex subset of $X$, and let $T: C \rightarrow C$ be a completely continuous asymptotically nonexpansive mapping with $\left\{k_{n}\right\}$ satisfying $k_{n} \geq 1, \sum_{n=1}^{\infty}\left(k_{n}^{r}-1\right)<\infty, r=\max \{2, p\}$. Then, for any $x_{1} \in C$, the sequence $\left\{x_{n}\right\}$ defined by (1.4), where $\left\{\alpha_{n}\right\}$ satisfies $a \leq \alpha_{n} \leq 1-a$ for all $n \geq 1$ and some $a>0$, converges strongly to some fixed point of $T$.

Theorem 1.2 Let $X$ be a uniformly convex Banach space, let $C$ be a nonempty bounded closed convex subset of $E$, and let $T: C \rightarrow C$ be a completely continuous asymptotically nonexpansive mapping with $\left\{k_{n}\right\}$ satisfying $k_{n} \geq 1, \sum_{n=1}^{\infty}\left(k_{n}^{r}-1\right)<\infty, r=\max \{2, p\}$. Then, for any $x_{1} \in C$, the sequence $\left\{x_{n}\right\}$ defined by (1.3), where $\left\{\alpha_{n}\right\},\left\{\beta_{n}\right\}$ satisfy $a \leq\left(1-\alpha_{n}\right),(1-$ $\left.\beta_{n}\right) \leq 1-a$ for all $n \geq 1$ and some $a>0$, converges strongly to some fixed point of $T$.

On the other hand, Kim [11] proved the following result which generalized Theorem 1 of Senter and Dotson [12].

Theorem 1.3 Let $X$ be a real uniformly convex Banach space, let $C$ be a nonempty closed convex subset of $X$, and let $T$ be a nonexpansive mapping of $C$ into itself satisfying condition (A) with $F(T) \neq \emptyset$. Suppose that for any $x_{1}$ in $C$, the sequence $\left\{x_{n}\right\}$ is defined by $x_{n+1}=$ $\left(1-\alpha_{n}\right) x_{n}+\alpha_{n}\left[\beta_{n} x_{n}+\left(1-\beta_{n}\right) T x_{n}\right]$, for all $n \geq 1$, where $\left\{\alpha_{n}\right\}$ and $\left\{\beta_{n}\right\}$ are sequences in $[0,1]$ such that $\sum_{n=1}^{\infty} \alpha_{n}\left(1-\alpha_{n}\right)=\infty$ and $\sum_{n=1}^{\infty} \beta_{n}<\infty$. Then $\left\{x_{n}\right\}$ converges strongly to some fixed point of $T$.

In this paper, we prove that if $T$ is a total asymptotically nonexpansive self-mapping satisfying condition (A), the iteration $\left\{x_{n}\right\}$ defined by (1.3) converges strongly to some fixed point of $T$, which generalizes the results due to Rhoades [1].

\section{Preliminaries}

Throughout this paper, we denote by $X$ a real Banach space. Let $C$ be a nonempty closed convex subset of $X$, and let $T$ be a mapping from $C$ into itself. Then we denote by $F(T)$ the 
set of all fixed points of $T$, i.e., $F(T)=\{x \in C: T x=x\}$. We also denote by $a \vee b:=\max \{a, b\}$. A Banach space $X$ is said to be uniformly convex if the modulus of convexity $\delta_{X}=\delta_{X}(\epsilon)$, $0<\epsilon \leq 2$, of $X$ defined by

$$
\delta_{X}(\epsilon)=\inf \left\{1-\frac{\|x+y\|}{2}: x, y \in X,\|x\| \leq 1,\|y\| \leq 1,\|x-y\| \geq \epsilon\right\}
$$

satisfies the inequality $\delta_{X}(\epsilon)>0$ for every $\epsilon \in(0,2]$. When $\left\{x_{n}\right\}$ is a sequence in $X$, then $x_{n} \rightarrow x$ will denote strong convergence of the sequence $\left\{x_{n}\right\}$ to $x$.

Definition 2.1 [12] A mapping $T: C \rightarrow C$ with $F(T) \neq \emptyset$ is said to satisfy condition (A) if there exists a nondecreasing function $f:[0, \infty) \rightarrow[0, \infty)$ with $f(0)=0$ and $f(r)>0$ for all $r \in(0, \infty)$ such that

$$
\|x-T x\| \geq f(d(x, F(T)))
$$

for all $x \in C$, where $d(x, F(T))=\inf _{z \in F(T)}\|x-z\|$.

\section{Strong convergence theorem}

We first begin with the following lemma.

Lemma 3.1 [13] Let $\left\{a_{n}\right\},\left\{b_{n}\right\}$ and $\left\{c_{n}\right\}$ be sequences of nonnegative real numbers such that $\sum_{n=1}^{\infty} b_{n}<\infty, \sum_{n=1}^{\infty} c_{n}<\infty$ and

$$
a_{n+1} \leq\left(1+b_{n}\right) a_{n}+c_{n}
$$

for all $n \geq 1$. Then $\lim _{n \rightarrow \infty} a_{n}$ exists.

Lemma 3.2 [14] Let $X$ be a uniformly convex Banach space. Let $x, y \in X$. If $\|x\| \leq 1,\|y\| \leq 1$ and $\|x-y\| \geq \epsilon>0$, then $\|\lambda x+(1-\lambda) y\| \leq 1-2 \lambda(1-\lambda) \delta(\epsilon)$ for $0 \leq \lambda \leq 1$.

Lemma 3.3 Let $C$ be a nonempty closed convex subset of a uniformly convex Banach space $X$, and let $T: C \rightarrow C$ be a TAN mapping with $F(T) \neq \emptyset$. Suppose that $\left\{c_{n}\right\},\left\{d_{n}\right\}$ and $\phi$ satisfy the following two conditions:

(I) $\exists \alpha, \beta>0$ such that $\phi(t) \leq \alpha$ for all $t \geq \beta$.

(II) $\sum_{n=1}^{\infty} c_{n}<\infty, \sum_{n=1}^{\infty} d_{n}<\infty$.

Suppose that the sequence $\left\{x_{n}\right\}$ is defined by (1.3). Then $\lim _{n \rightarrow \infty}\left\|x_{n}-z\right\|$ exists for any $z \in F(T)$.

Proof For any $z \in F(T)$, we set

$$
M:=1 \vee \phi(\beta)<\infty
$$

From (I) and strict increasing of $\phi$, we obtain

$$
\phi(t) \leq \phi(\beta)+\alpha t, \quad t \geq 0 .
$$


By using (3.1), we have

$$
\begin{aligned}
\left\|T^{n} x_{n}-z\right\| & \leq\left\|x_{n}-z\right\|+c_{n} \phi\left(\left\|x_{n}-z\right\|\right)+d_{n} \\
& \leq\left\|x_{n}-z\right\|+c_{n}\left\{\phi(\beta)+\alpha\left\|x_{n}-z\right\|\right\}+d_{n} \\
& \leq\left(1+\alpha c_{n}\right)\left\|x_{n}-z\right\|+\kappa_{n} M,
\end{aligned}
$$

where $\kappa_{n}=c_{n}+d_{n}$ and $\sum_{n=1}^{\infty} \kappa_{n}<\infty$. Since

$$
\begin{aligned}
\left\|y_{n}-z\right\| & =\left\|\beta_{n} T^{n} x_{n}+\left(1-\beta_{n}\right) x_{n}-z\right\| \\
& \leq \beta_{n}\left\|T^{n} x_{n}-z\right\|+\left(1-\beta_{n}\right)\left\|x_{n}-z\right\| \\
& \leq \beta_{n}\left\{\left(1+\alpha c_{n}\right)\left\|x_{n}-z\right\|+\kappa_{n} M\right\}+\left(1-\beta_{n}\right)\left\|x_{n}-z\right\| \\
& \leq\left(1+\alpha c_{n}\right)\left\|x_{n}-z\right\|+\kappa_{n} M,
\end{aligned}
$$

and thus

$$
\begin{aligned}
& \left\|y_{n}-z\right\|+c_{n} \phi\left(\left\|y_{n}-z\right\|\right) \\
& \quad \leq\left(1+\alpha c_{n}\right)\left\|x_{n}-z\right\|+\kappa_{n} M+c_{n}\left\{\phi(\beta)+\alpha\left\|y_{n}-z\right\|\right\} \\
& \quad \leq\left(1+\alpha c_{n}\right)\left\|x_{n}-z\right\|+\kappa_{n} M+c_{n} \phi(\beta)+\alpha c_{n}\left(1+\alpha c_{n}\right)\left\|x_{n}-z\right\|+\alpha c_{n} \kappa_{n} M \\
& \quad \leq\left(1+\sigma_{n}\right)\left\|x_{n}-z\right\|+\delta_{n} M,
\end{aligned}
$$

where $\sigma_{n}=2 \alpha c_{n}+\alpha^{2} c_{n}^{2}, \delta_{n}=\kappa_{n}+c_{n}+\alpha c_{n} \kappa_{n}, \sum_{n=1}^{\infty} \sigma_{n}<\infty$ and $\sum_{n=1}^{\infty} \delta_{n}<\infty$. So, we have

$$
\begin{aligned}
\left\|T^{n} y_{n}-z\right\| & \leq\left\|y_{n}-z\right\|+c_{n} \phi\left(\left\|y_{n}-z\right\|\right)+d_{n} \\
& \leq\left(1+\sigma_{n}\right)\left\|x_{n}-z\right\|+\delta_{n} M+d_{n} \\
& \leq\left(1+\sigma_{n}\right)\left\|x_{n}-z\right\|+\eta_{n} M,
\end{aligned}
$$

where $\eta_{n}=\delta_{n}+d_{n}$ and $\sum_{n=1}^{\infty} \eta_{n}<\infty$. Hence

$$
\begin{aligned}
\left\|x_{n+1}-z\right\| & =\left\|\left(1-\alpha_{n}\right) x_{n}+\alpha_{n} T^{n} y_{n}-z\right\| \\
& \leq\left(1-\alpha_{n}\right)\left\|x_{n}-z\right\|+\alpha_{n}\left\|T^{n} y_{n}-z\right\| \\
& \leq\left(1-\alpha_{n}\right)\left\|x_{n}-z\right\|+\alpha_{n}\left\{\left(1+\sigma_{n}\right)\left\|x_{n}-z\right\|+\eta_{n} M\right\} \\
& \leq\left(1+\sigma_{n}\right)\left\|x_{n}-z\right\|+\eta_{n} M .
\end{aligned}
$$

By Lemma 3.1, we see that $\lim _{n \rightarrow \infty}\left\|x_{n}-z\right\|$ exists.

Theorem 3.4 Let $X$ be a uniformly convex Banach space, and let $C$ be a nonempty closed convex subset of $X$. Let $T: C \rightarrow C$ be a uniformly continuous and TAN mapping with $F(T) \neq \emptyset$. Suppose that $\left\{c_{n}\right\},\left\{d_{n}\right\}$ and $\phi$ satisfy the following two conditions:

(I) $\exists \alpha, \beta>0$ such that $\phi(t) \leq \alpha$ for all $t \geq \beta$.

(II) $\sum_{n=1}^{\infty} c_{n}<\infty, \sum_{n=1}^{\infty} d_{n}<\infty$. 
Suppose that for any $x_{1}$ in $C$, the sequence $\left\{x_{n}\right\}$ defined by (1.3) satisfies $\sum_{n=1}^{\infty} \alpha_{n}\left(1-\alpha_{n}\right)=\infty$ and $\lim \beta_{n}=0$. Then $\left\{x_{n}\right\}$ converges strongly to some fixed point of $T$.

Proof For any $z \in F(T)$, by Lemma 3.3, $\left\{x_{n}\right\}$ is bounded. We set

$$
M:=1 \vee \phi(\beta) \vee \sup _{n \geq 1}\left\|x_{n}-z\right\|<\infty .
$$

By Lemma 3.3, we see that $\lim _{n \rightarrow \infty}\left\|x_{n}-z\right\|(\equiv r)$ exists. Without loss of generality, we assume $r>0$. As in the proof of Lemma 3.3, we obtain

$$
\begin{aligned}
\left\|T^{n} y_{n}-z\right\| & \leq\left(1+\sigma_{n}\right)\left\|x_{n}-z\right\|+\eta_{n} M \\
& \leq\left\|x_{n}-z\right\|+v_{n} M,
\end{aligned}
$$

where $v_{n}=\sigma_{n}+\eta_{n}$ and $\sum_{n=1}^{\infty} v_{n}<\infty$. By using Lemma 3.2 and Takahashi [15], we obtain

$$
\begin{aligned}
\left\|x_{n+1}-z\right\| & =\left\|\left(1-\alpha_{n}\right) x_{n}+\alpha_{n} T^{n} y_{n}-z\right\| \\
& =\left\|\left(1-\alpha_{n}\right)\left(x_{n}-z\right)+\alpha_{n}\left(T^{n} y_{n}-z\right)\right\| \\
& \leq\left(\left\|x_{n}-z\right\|+v_{n} M\right)\left[1-2 \alpha_{n}\left(1-\alpha_{n}\right) \delta_{X}\left(\frac{\left\|T^{n} y_{n}-x_{n}\right\|}{\left\|x_{n}-z\right\|+v_{n} M}\right)\right] .
\end{aligned}
$$

Hence we obtain

$$
\begin{aligned}
& 2 \alpha_{n}\left(1-\alpha_{n}\right)\left(\left\|x_{n}-z\right\|+v_{n} M\right) \delta_{X}\left(\frac{\left\|T^{n} y_{n}-x_{n}\right\|}{\left\|x_{n}-z\right\|+v_{n} M}\right) \\
& \quad \leq\left\|x_{n}-z\right\|-\left\|x_{n+1}-z\right\|+v_{n} M .
\end{aligned}
$$

Thus

$$
2 \alpha_{n}\left(1-\alpha_{n}\right)\left(\left\|x_{n}-z\right\|+v_{n} M\right) \delta_{X}\left(\frac{\left\|T^{n} y_{n}-x_{n}\right\|}{\left\|x_{n}-z\right\|+v_{n} M}\right)<\infty .
$$

Since $\delta_{X}$ is strictly increasing, continuous and $\sum_{n=1}^{\infty} \alpha_{n}\left(1-\alpha_{n}\right)=\infty$, we obtain

$$
\liminf _{n \rightarrow \infty}\left\|T^{n} y_{n}-x_{n}\right\|=0
$$

By using (3.1) in the proof of Lemma 3.3, we have

$$
\begin{aligned}
\left\|T^{n-1} x_{n-1}-z\right\| & \leq\left\|x_{n-1}-z\right\|+c_{n-1} \phi\left(\left\|x_{n-1}-z\right\|\right)+d_{n-1} \\
& \leq\left\|x_{n-1}-z\right\|+c_{n-1}\left\{\phi(\beta)+\alpha\left\|x_{n-1}-z\right\|\right\}+d_{n-1} \\
& \leq\left(1+\alpha c_{n-1}\right)\left\|x_{n-1}-z\right\|+\rho_{n-1} M,
\end{aligned}
$$

where $\rho_{n-1}=c_{n-1}+d_{n-1}$ and $\sum_{n=2}^{\infty} \rho_{n-1}<\infty$. Thus

$$
\begin{aligned}
\left\|y_{n-1}-z\right\| & =\left\|\beta_{n-1} T^{n-1} x_{n-1}+\left(1-\beta_{n-1}\right) x_{n-1}-z\right\| \\
& \leq \beta_{n-1}\left\|T^{n-1} x_{n-1}-z\right\|+\left(1-\beta_{n-1}\right)\left\|x_{n-1}-z\right\|
\end{aligned}
$$




$$
\begin{aligned}
& \leq \beta_{n-1}\left\{\left(1+\alpha c_{n-1}\right)\left\|x_{n-1}-z\right\|+\rho_{n-1} M\right\}+\left(1-\beta_{n-1}\right)\left\|x_{n-1}-z\right\| \\
& \leq\left(1+\alpha c_{n-1}\right)\left\|x_{n-1}-z\right\|+\rho_{n-1} M,
\end{aligned}
$$

and hence

$$
\begin{aligned}
\left\|y_{n-1}-z\right\|+c_{n-1} \phi\left(\left\|y_{n-1}-z\right\|\right) & \\
\leq & \left(1+\alpha c_{n-1}\right)\left\|x_{n-1}-z\right\|+\rho_{n-1} M+c_{n-1}\left\{\phi(\beta)+\alpha\left\|y_{n-1}-z\right\|\right\} \\
\leq & \left(1+\alpha c_{n-1}\right)\left\|x_{n-1}-z\right\|+\rho_{n-1} M+c_{n-1} \phi(\beta)+\alpha c_{n-1}\left(1+\alpha c_{n-1}\right)\left\|x_{n-1}-z\right\| \\
& \quad+\alpha c_{n-1} \rho_{n-1} M \\
\leq & \left(1+\mu_{n-1}\right)\left\|x_{n-1}-z\right\|+\varphi_{n-1} M,
\end{aligned}
$$

where $\mu_{n-1}=2 \alpha c_{n-1}+\alpha^{2} c_{n-1}^{2}, \varphi_{n-1}=\rho_{n-1}+c_{n-1}+\alpha c_{n-1} \rho_{n-1}, \quad \sum_{n=2}^{\infty} \mu_{n-1}<\infty$ and $\sum_{n=2}^{\infty} \varphi_{n-1}<\infty$. So, we have

$$
\begin{aligned}
\left\|T^{n-1} y_{n-1}-z\right\| & \leq\left\|y_{n-1}-z\right\|+c_{n-1} \phi\left(\left\|y_{n-1}-z\right\|\right)+d_{n-1} \\
& \leq\left(1+\mu_{n-1}\right)\left\|x_{n-1}-z\right\|+\varphi_{n-1} M+d_{n-1} \\
& \leq\left\|x_{n-1}-z\right\|+\omega_{n-1} M
\end{aligned}
$$

where $\omega_{n-1}=\mu_{n-1}+\varphi_{n-1}+d_{n-1}$ and $\sum_{n=2}^{\infty} \omega_{n-1}<\infty$. By using Lemma 3.2 and Takahashi [15], we obtain

$$
\begin{aligned}
\left\|x_{n}-z\right\| & =\left\|\left(1-\alpha_{n-1}\right) x_{n-1}+\alpha_{n-1} T^{n-1} y_{n-1}-z\right\| \\
& =\left\|\left(1-\alpha_{n-1}\right)\left(x_{n-1}-z\right)+\alpha_{n-1}\left(T^{n-1} y_{n-1}-z\right)\right\| \\
& \leq\left(\left\|x_{n-1}-z\right\|+\omega_{n-1} M\right)\left[1-2 \alpha_{n}\left(1-\alpha_{n}\right) \delta_{X}\left(\frac{\left\|T^{n-1} y_{n-1}-x_{n-1}\right\|}{\left\|x_{n-1}-z\right\|+\omega_{n-1} M}\right)\right] .
\end{aligned}
$$

By the same method as above, we obtain

$$
\liminf _{n \rightarrow \infty}\left\|T^{n-1} y_{n-1}-x_{n-1}\right\|=0
$$

Since $\left\{x_{n}\right\}$ is bounded and $T$ is a TAN mapping, we obtain

$$
\begin{aligned}
\left\|y_{n}-x_{n}\right\| & =\left\|\beta_{n} T^{n} x_{n}+\left(1-\beta_{n}\right) x_{n}-x_{n}\right\| \\
& \leq \beta_{n}\left\|T^{n} x_{n}-x_{n}\right\| \\
& \leq \beta_{n} M^{\prime}
\end{aligned}
$$

where $M^{\prime}=\sup _{n \geq 1}\left\|T^{n} x_{n}-x_{n}\right\|<\infty$. By using $\lim \beta_{n}=0$, we have

$$
\lim _{n \rightarrow \infty}\left\|x_{n}-y_{n}\right\|=0
$$

Since

$$
\left\|T^{n} y_{n}-y_{n}\right\| \leq\left\|T^{n} y_{n}-x_{n}\right\|+\left\|x_{n}-y_{n}\right\|,
$$


by (3.2) and (3.4), we obtain

$$
\liminf _{n \rightarrow \infty}\left\|T^{n} y_{n}-y_{n}\right\|=0 .
$$

By using (3.3) and (3.4), we obtain

$$
\liminf _{n \rightarrow \infty}\left\|T^{n-1} y_{n-1}-y_{n-1}\right\|=0 .
$$

Since

$$
\begin{aligned}
\left\|T^{n-1} x_{n-1}-x_{n-1}\right\| \leq & \left\|T^{n-1} x_{n-1}-T^{n-1} y_{n-1}\right\|+\left\|T^{n-1} y_{n-1}-x_{n-1}\right\| \\
\leq & \left\|x_{n-1}-y_{n-1}\right\|+c_{n-1} \phi\left(\left\|x_{n-1}-y_{n-1}\right\|\right)+d_{n-1} \\
& +\left\|T^{n-1} y_{n-1}-x_{n-1}\right\|,
\end{aligned}
$$

by using (3.3) and (3.4), we have

$$
\liminf _{n \rightarrow \infty}\left\|T^{n-1} x_{n-1}-x_{n-1}\right\|=0
$$

Since

$$
\begin{aligned}
\left\|x_{n}-x_{n-1}\right\| & =\left\|\left(1-\alpha_{n-1}\right) x_{n-1}+\alpha_{n-1} T^{n-1} y_{n-1}-x_{n-1}\right\| \\
& =\alpha_{n-1}\left\|T^{n-1} y_{n-1}-x_{n-1}\right\| \\
& \leq\left\|T^{n-1} y_{n-1}-y_{n-1}\right\|+\left\|y_{n-1}-x_{n-1}\right\|,
\end{aligned}
$$

by (3.4) and (3.6), we get

$$
\liminf _{n \rightarrow \infty}\left\|x_{n}-x_{n-1}\right\|=0
$$

From

$$
\begin{aligned}
\left\|T^{n-1} x_{n}-x_{n}\right\| & \leq\left\|T^{n-1} x_{n}-T^{n-1} x_{n-1}\right\|+\left\|T^{n-1} x_{n-1}-x_{n-1}\right\|+\left\|x_{n-1}-x_{n}\right\| \\
& \leq 2\left\|x_{n}-x_{n-1}\right\|+c_{n-1} \phi\left(\left\|x_{n}-x_{n-1}\right\|\right)+d_{n-1}+\left\|T^{n-1} x_{n-1}-x_{n-1}\right\|,
\end{aligned}
$$

by (3.7) and (3.8), we obtain

$$
\liminf _{n \rightarrow \infty}\left\|T^{n-1} x_{n}-x_{n}\right\|=0 .
$$

Since

$$
\begin{aligned}
& \left\|x_{n}-T x_{n}\right\| \\
& \quad \leq\left\|x_{n}-y_{n}\right\|+\left\|y_{n}-T^{n} y_{n}\right\|+\left\|T^{n} y_{n}-T^{n} x_{n}\right\|+\left\|T^{n} x_{n}-T x_{n}\right\| \\
& \quad \leq\left\|y_{n}-T^{n} y_{n}\right\|+2\left\|x_{n}-y_{n}\right\|+c_{n} \phi\left(\left\|x_{n}-y_{n}\right\|\right)+d_{n}+\left\|T^{n} x_{n}-T x_{n}\right\|
\end{aligned}
$$


and by the uniform continuity of $T,(3.4),(3.5)$ and (3.9), we have

$$
\liminf _{n \rightarrow \infty}\left\|x_{n}-T x_{n}\right\|=0
$$

By using condition (A), we obtain

$$
f\left(d\left(x_{n}, F(T)\right)\right) \leq\left\|x_{n}-T x_{n}\right\|
$$

for all $n \geq 1$. As in the proof of Lemma 3.3, we obtain

$$
\left\|x_{n+1}-z\right\| \leq\left(1+\sigma_{n}\right)\left\|x_{n}-z\right\|+\eta_{n} M
$$

Thus

$$
\inf _{z \in F(T)}\left\|x_{n+1}-z\right\| \leq\left(1+\sigma_{n}\right) \inf _{z \in F(T)}\left\|x_{n}-z\right\|+\eta_{n} M
$$

By using Lemma 3.1, we see that $\lim _{n \rightarrow \infty} d\left(x_{n}, F(T)\right)(\equiv c)$ exists. We first claim that $\lim _{n \rightarrow \infty} d\left(x_{n}, F(T)\right)=0$. In fact, assume that $c=\lim _{n \rightarrow \infty} d\left(x_{n}, F(T)\right)>0$. Then we can choose $n_{0} \in \mathbb{N}$ such that $0<\frac{c}{2}<d\left(x_{n}, F(T)\right)$ for all $n \geq n_{0}$. By using condition (A), (3.10) and (3.11), we obtain

$$
0<f\left(\frac{c}{2}\right) \leq f\left(d\left(x_{n_{i}}, F(T)\right)\right) \leq\left\|x_{n_{i}}-T x_{n_{i}}\right\| \rightarrow 0
$$

as $i \rightarrow \infty$. This is a contradiction. So, we obtain $c=0$. Next, we claim that $\left\{x_{n}\right\}$ is a Cauchy sequence. Since $\sum_{n=1}^{\infty} \sigma_{n}<\infty$, we obtain $\prod_{n=1}^{\infty}\left(1+\sigma_{n}\right):=U<\infty$. Let $\epsilon>0$ be given. Since $\lim _{n \rightarrow \infty} d\left(x_{n}, F(T)\right)=0$ and $\sum_{n=1}^{\infty} \eta_{n}<\infty$, there exists $n_{0} \in \mathbb{N}$ such that for all $n \geq n_{0}$, we obtain

$$
d\left(x_{n}, F(T)\right)<\frac{\epsilon}{4 U+4} \quad \text { and } \quad \sum_{i=n_{0}}^{\infty} \eta_{i}<\frac{\epsilon}{4 M}
$$

Let $n, m \geq n_{0}$ and $p \in F(T)$. Then, by (3.12), we obtain

$$
\begin{aligned}
\left\|x_{n}-x_{m}\right\| & \leq\left\|x_{n}-p\right\|+\left\|x_{m}-p\right\| \\
& \leq \prod_{i=n_{0}}^{n-1}\left(1+\sigma_{i}\right)\left\|x_{n_{0}}-p\right\|+M \sum_{i=n_{0}}^{n-1} \eta_{i}+\prod_{i=n_{0}}^{m-1}\left(1+\sigma_{i}\right)\left\|x_{n_{0}}-p\right\|+M \sum_{i=n_{0}}^{m-1} \eta_{i} \\
& \leq 2\left[\prod_{i=n_{0}}^{\infty}\left(1+\sigma_{i}\right)\left\|x_{n_{0}}-p\right\|+M \sum_{i=n_{0}}^{\infty} \eta_{i}\right] .
\end{aligned}
$$

Taking the infimum over all $p \in F(T)$ on both sides and by (3.13), we obtain

$$
\begin{aligned}
\left\|x_{n}-x_{m}\right\| & \leq 2\left[\prod_{i=n_{0}}^{\infty}\left(1+\sigma_{i}\right) d\left(x_{n_{0}}, F(T)\right)+M \sum_{i=n_{0}}^{\infty} \eta_{i}\right] \\
& <2\left[(U+1) \frac{\epsilon}{4 U+4}+M \frac{\epsilon}{4 M}\right]=\epsilon
\end{aligned}
$$


for all $n, m \geq n_{0}$. This implies that $\left\{x_{n}\right\}$ is a Cauchy sequence. Let $\lim _{n \rightarrow \infty} x_{n}=q$. Then $d(q, F(T))=0$. Since $F(T)$ is closed, we obtain $q \in F(T)$. Hence $\left\{x_{n}\right\}$ converges strongly to some fixed point of $T$.

Remark 3.5 If $T: C \rightarrow C$ is completely continuous, then it satisfies demicompact and, if $T$ is continuous and demicompact, it satisfies condition (A); see Senter and Dotson [12].

Remark 3.6 If $\left\{\alpha_{n}\right\}$ is bounded away from both 0 and 1, i.e., $a \leq \alpha_{n} \leq b$ for all $n \geq 1$ and some $a, b \in(0,1)$, then $\sum_{n=1}^{\infty} \alpha_{n}\left(1-\alpha_{n}\right)=\infty$ and $\lim _{n \rightarrow \infty} \beta_{n}=0$ hold. However, the converse is not true. For example, consider $\alpha_{n}=\frac{1}{n}$.

We give an example of a mapping $T: C \rightarrow C$ which satisfies all the assumptions of $T$ in Theorem 3.4, i.e., $T: C \rightarrow C$ is a uniformly continuous mapping with $F(T) \neq \emptyset$ which is TAN on $C$, not Lipschitzian and hence not asymptotically nonexpansive.

Example 3.7 Let $X:=\mathbb{R}$ and $C:=[0,2]$. Define $T: C \rightarrow C$ by

$$
T x= \begin{cases}1, & x \in[0,1] \\ \frac{1}{\sqrt{3}} \sqrt{4-x^{2}}, & x \in[1,2]\end{cases}
$$

Note that $T^{n} x=1$ for all $x \in C$ and $n \geq 2$ and $F(T)=\{1\}$. Clearly, $T$ is both uniformly continuous and TAN on $C$. We show that $T$ satisfies condition (A). In fact, if $x \in[0,1]$, then $|x-1|=|x-T x|$. Similarly, if $x \in[1,2]$, then

$$
|x-1|=x-1 \leq x-\frac{1}{\sqrt{3}} \sqrt{4-x^{2}}=|x-T x| .
$$

So, we get $d(x, F(T))=|x-1| \leq|x-T x|$ for all $x \in C$. But $T$ is not Lipschitzian. Indeed, suppose not, i.e., there exists $L>0$ such that

$$
|T x-T y| \leq L|x-y|
$$

for all $x, y \in C$. If we take $x=2-\frac{1}{3(L+1)^{2}}>1$ and $y=2$, then

$$
\frac{1}{\sqrt{3}} \sqrt{4-x^{2}} \leq L(2-x) \Leftrightarrow \frac{1}{3 L^{2}} \leq \frac{2-x}{2+x}=\frac{1}{12 L^{2}+24 L+1} .
$$

This is a contradiction.

Competing interests

The author declares that they have no competing interests.

\section{Acknowledgements}

The author would like to express their sincere appreciation to the anonymous referees for useful suggestions which improved the contents of this manuscript. 


\section{References}

1. Rhoades, BE: Fixed point iterations for certain nonlinear mappings. J. Math. Anal. Appl. 183, 118-120 (1994)

2. Goebel, K, Kirk, WA: A fixed point theorem for asymptotically nonexpansive mappings. Proc. Am. Math. Soc. 35 , 171-174 (1972)

3. Alber, $\mathrm{Yl}$, Chidume, $\mathrm{CE}$, Zegeye, $\mathrm{H}$ : Approximating fixed points of total asymptotically nonexpansive mappings. Fixed Point Theory Appl. 2006, Article ID 10673 (2006)

4. Chidume, CE, Ofoedu, EU: Approximation of common fixed points for finite families of total asymptotically nonexpansive mappings. J. Math. Anal. Appl. 333(1), 128-141 (2007)

5. Chidume, CE, Ofoedu, EU: A new iteration process for approximation of common fixed points for finite families of total asymptotically nonexpansive mappings. Int. J. Math. Math. Sci. 2009, Article ID 615107 (2009)

6. Kim, GE: Approximating common fixed points for total asymptotically nonexpansive mappings. J. Appl. Math. Inform. 30, 71-82 (2012)

7. Kim, GE, Kim, TH: Strong convergence theorems of total asymptotically nonexpansive mappings. In: Proceedings of the 7th International Conference on Nonlinear Analysis and Convex Analysis, pp. 197-208 (2011)

8. Schu, J: Iterative construction of fixed points of asymptotically nonexpansive mappings. J. Math. Anal. Appl. 158, 407-413 (1991)

9. Ishikawa, S: Fixed points by a new iteration method. Proc. Am. Math. Soc. 44, 147-150 (1974)

10. Mann, WR: Mean value methods in iteration. Proc. Am. Math. Soc. 4, 506-510 (1953)

11. Kim, GE: Weak and strong convergence of the modified Mann iteration process for nonexpansive mappings. J. Nonlinear Convex Anal. 13(3), 449-457 (2012)

12. Senter, HF, Dotson, WG: Approximating fixed points of nonexpansive mappings. Proc. Am. Math. Soc. 44, 375-380 (1974)

13. Qihou, L: Iterative sequences for asymptotically quasi-nonexpansive mappings with error member. J. Math. Anal. Appl. 259, 18-24 (2001)

14. Groetsch, CW: A note on segmenting Mann iterates. J. Math. Anal. Appl. 40, 369-372 (1972)

15. Takahashi, W: Nonlinear Functional Analysis. Yokohama-Publishers, Yokohama (2000)

10.1186/1687-1812-2013-302

Cite this article as: Kim: Strong convergence to a fixed point of a total asymptotically nonexpansive mapping. Fixed Point Theory and Applications 2013, 2013:302

\section{Submit your manuscript to a SpringerOpen ${ }^{\circ}$ journal and benefit from:}

- Convenient online submission

- Rigorous peer review

- Immediate publication on acceptance

Open access: articles freely available online

- High visibility within the field

- Retaining the copyright to your article 